\title{
Impact of extra-corporeal life support (ECLS) cannulation strategy on outcome after durable mechanical circulation support system implantation on behalf of durable MCS after ECLS Study Group
}

\author{
Diyar Saeed $^{1,2}$, Evgenij Potapov ${ }^{3,4}$, Antonio Loforte ${ }^{5}$, Michiel Morshuis ${ }^{6}$, David Schibilsky ${ }^{7}$, \\ Daniel Zimpfer ${ }^{8}$, Julia Riebandt ${ }^{8}$, Federico Pappalardo ${ }^{9}$, Matteo Attisani ${ }^{10}$, Mauro Rinaldi ${ }^{10}$, \\ Davide Pacini $^{5}$, Assad Haneya ${ }^{11}$, Faiz Ramjankhan ${ }^{12}$, Dirk W. Donker ${ }^{12}$, Ulrich P. Jorde ${ }^{13}$, Wolfgang Otto ${ }^{1}$, \\ Julia Stein ${ }^{3}$, Dmytro Tsyganenko ${ }^{3}$, Ameen Al-Naamani ${ }^{1}$, Radi Wieloch ${ }^{2}$, Rafael Ayala ${ }^{7}$, Jochen Cremer ${ }^{11}$, \\ Michael Borger ${ }^{1}$, Artur Lichtenberg ${ }^{2}$, Jan Gummert ${ }^{6}$
}

${ }^{1}$ Department of Cardiac surgery, Leipzig Heart Center, Leipzig, Germany; ${ }^{2}$ Department for Cardiac Surgery, Duesseldorf University Hospital, Duesseldorf, Germany; ${ }^{3}$ Department of Cardiac Surgery, German Heart Center Berlin, Berlin, Germany; ${ }^{4}$ DZHK (German Center for Cardiovascular Research), Partner Site Berlin, Berlin, Germany; ${ }^{5}$ Division of Cardiac Surgery, IRCCS Azienda Ospedaliero-Universitaria di Bologna, Bologna, Italy; ${ }^{6}$ Department of Cardiovascular and Thoracic Surgery, Heart and diabetes Center NRW, Bad Oeynhausen, Germany; ${ }^{7}$ Department of Cardiac and Vascular Surgery, Freiburg University, Freiburg, Germany; ${ }^{8}$ Department of Cardiac Surgery, Medical University Vienna, Vienna, Austria; ${ }^{9}$ Advanced Heart Failure and Mechanical Circulatory Support Program, San Raffaele Hospital, Vita Salute University, Milan, Italy; ${ }^{10}$ Department of Cardiac Surgery, University of Turin, Turin, Italy; ${ }^{11}$ Department of Cardiac Surgery, University Hospital Schleswig Holstein, Campus Kiel, Kiel, Germany; ${ }^{12}$ Department of Cardiothoracic Surgery, University Medical Center Utrecht, Utrecht, The Netherland; ${ }^{13}$ Department of Medicine, Montefiore Medical Center, Bronx, NY, USA

Correspondence to: Diyar Saeed, MD, PhD. University Department for Cardiac Surgery, Leipzig Heart Center, Strümpellstr. 39 , 04289 Leipzig, Germany. Email: diyar.saeed@helios-gesundheit.de.

Background: The literature on outcomes of patients requiring durable mechanical circulatory support (MCS) after extra-corporeal life support (ECLS) is limited. The aim of this study was to investigate the impact of preoperative ECLS cannulation on postoperative outcome after durable MCS implantation.

Methods: The durable MCS after ECLS registry is a multicenter retrospective study that gathered data on consecutive patients who underwent durable MCS implantation after ECLS between January 2010 and August 2018 in eleven high volume European centers. Patients who underwent the implantation of total artificial heart, pulsatile pumps, or first-generation pumps after ECLS were excluded from the analysis. The remaining patients were divided into two groups; central ECLS group (cECLS) and peripheral ECLS group (pECLS). A 1:1 propensity score analysis was performed to identify two matched groups. The outcome of these two groups was compared.

Results: A total of 531 durable MCS after ECLS were implanted during this period. The ECLS cannulation site was peripheral in $87 \%(n=462)$ and central in $13 \%(n=69)$ of the patients. After excluding pulsatile pumps and total artificial heart patients, a total of 494 patients remained (pECLS $=434$ patients, cECLS $=60$ patients). A 1:1 propensity score analysis resulted in 2 matched groups (each 55 patients) with median age of 54 years (48-60 years) in cECLS group and 54 years (43-60 years) in pECLS group. HeartWare HVAD (Medtronic, Minneapolis, MN) was implanted in the majority of the patients (cECLS $=71 \%$ vs. pECLS $=76 \%, \mathrm{P}=0.67)$. All postoperative morbidities were comparable between the groups. The thirty-day, one year and long-term survival was comparable between the groups $(\mathrm{P}=0.73)$.

Conclusions: The cannulation strategy of ECLS appears to have no impact on the post-operative outcome after durable MCS implantation.

Keywords: Mechanical circulatory support (MCS); extra-corporeal life support (ECLS); cannulation strategy; outcome; ventricular assist device

(C) Annals of Cardiothoracic Surgery. All rights reserved. Ann Cardiothorac Surg 2021;10(3):353-363 I http://dx.doi.org/10.21037/acs-2020-cfmcs-251 
Submitted Dec 21, 2020. Accepted for publication Mar 29, 2021.

doi: $10.21037 /$ acs-2020-cfmcs-251

View this article at: http://dx.doi.org/10.21037/acs-2020-cfmcs-251

\section{Introduction}

Mechanical circulatory support (MCS) systems, in particular, extra-corporeal life support (ECLS), have become a widely accepted therapy option for patients in cardiogenic shock (1). The application of ECLS for immediate resuscitation with the potential of end organ function recovery is a useful strategy to improve survival of this otherwise extremely sick patient population. After ECLS implantation, the primary aim remains patient stabilization and weaning from ECLS. However, some patients take a different path and either expire on ECLS due to multiple organ failure and/or neurological deficit, or need to be considered for a durable MCS. The decision to implant a durable MCS in a patient with ECLS is very challenging and the implantation threshold may vary between institutions $(2,3)$. Our group recently published the largest series on durable MCS patients bridged with ECLS (4). We were able to identify several survival predictors. An app "Durable MCS after ECLS calculator" was introduced that aids future patient selection and helps to avoid unnecessary resource utilization (4).

The implantation of ECLS is usually on an emergency basis for cardiogenic shock patients. The implantation may be performed using either a central approach with the oxygenated blood from ECLS returning to the aorta/subclavian artery or a peripheral approach with oxygenated blood returned through the femoral artery. There are several studies investigating the impact of ECLS cannulation approach on various post implant morbidities and outcome after ECLS implantation $(5,6)$ However, we are not aware of any study that specifically investigates the impact of preoperative (prior to durable MCS implantation) ECLS cannulation strategy on postoperative morbidities and outcome after durable MCS implantation. Therefore, the primary objective of this study was to investigate whether preoperative ECLS cannulation strategy has any impact on the outcome after durable MCS implantation using data from the durable MCS after ECLS registry. We hypothesize that the more physiological form of support, central ECLS, may be associated with lower postoperative morbidities after durable MCS implantation.

\section{Methods}

\section{Patient population}

The durable MCS after ECLS registry is a multicenter retrospective study that gathered data on consecutive patients who underwent durable MCS implantation directly after ECLS between January 2010 and August 2018 in eleven high volume European centers. The primary aim after ECLS implantation was to wean the patient off mechanical support. Patients who did not meet the weaning criteria were considered for durable MCS after adequate neurological evaluation. There was no specific protocol when and how to proceed with durable MCS therapy. All perioperative data and postoperative complications were considered. The Interagency Registry for Mechanically Assisted Circulatory Support (INTERMACS) definitions were used for postoperative complications except for right ventricular failure, which was considered only if mechanical support of the right ventricle was necessary.

For this study, all patients who underwent the implantation of total artificial heart, pulsatile pumps, or earlier generation pumps after ECLS were excluded from the analysis. Therefore, only patients who were supported with HeartWare HVAD (Medtronic, Minneapolis, MN), HeartMate II (Abbott, Abbott Park, Ill) or HeartMate III (Abbott, Abbott Park, Ill) were included. The patients were divided into two groups; central ECLS group (cECLS) and peripheral ECLS group (pECLS). A 1:1 propensity score analysis was then performed to identify two matched groups. The outcome of these two groups was analyzed and compared. The study protocol was approved by the Individual Health Research Ethics Boards.

\section{Surgical techniques}

\section{ECLS implantation}

The ECLS implantation at each institution was performed on an emergency basis in cardiogenic shock patients for various reasons. Peripheral ECLS approach was predominantly performed through femoral vein and arteries. Distal leg perfusion cannula was exclusively used in 
pECLS patients. The cannulation was either percutaneous or through surgical cut down. The arterial cannulation strategy for central ECLS approach was either through direct cannulation of the aorta, graft anastomosis to the aorta, or graft anastomosis to the subclavian artery. The venous cannulation of central ECLS was predominately percutaneous using the femoral vein.

\section{Durable MCS implantation}

The durable MCS was implanted either in a standard fashion through a median sternotomy or using less invasive techniques. The outflow graft was connected to the ascending aorta in all cases. Some of the patients were operated on ECLS. However, the cardiopulmonary bypass machine was used at the time of implant for other patients, either due to the necessity of concomitant procedures or surgeon preference.

\section{Statistical analysis}

Continuous study variables were evaluated for both normal distribution and outlier activity. They were reported as mean with standard deviation if normally distributed, or as median with the interquartile range otherwise. For categorical data, the frequencies are given. Statistical tests were performed according to type, normality, and scedasticity of data with Welch two-sample $t$-test, Wilcoxon signed-rank test, or $\chi^{2}$ test. A 5 -fold multiple imputation was applied to address missing values in risk factors. Patients in the cECLS group were compared to patients in the pECLS group. As these two groups were not randomized, a propensity score analysis was computed with univariate analysis assessing a predefined set of preoperative risk factors. The 1:1 nearest neighbor propensity score matching with a caliper of 0.2 was applied onto relevant baseline variables stratifying 55 patients into each group (Figure 1). Kaplan-Meier estimates were calculated to describe overall survival in the matched pECLS and cECLS groups with the date of MCS implantation as the starting point. Comparison of the matched cECLS versus pECLS groups was performed using log-rank test. For statistical calculations and graphics, we used R software, Version 4.0.3 (R Core Team 2018. R: A Language and Environment for Statistical Computing. R Foundation for Statistical Computing, Vienna, Austria) with the packages mice, MatchIt and tidyverse.

\section{Results}

A total of 531 durable MCS on VA-ECMO with average age of $53 \pm 12$ years were implanted between January 2010 and August 2018. Ischemic cardiomyopathy was the dominant reason for cardiogenic shock with 300 patients (57\%). The ECLS cannulation was peripheral in $462(87 \%)$ of patients and central in 69 patients (13\%). Up to 173 (33\%) patients had history of cardiopulmonary resuscitation (CPR) prior to ECLS implantation. After excluding pulsatile pumps, first generation pumps and total artificial heart patients, a total of 494 patients remained (pECLS $=434$ patients, cECLS $=60$ patients). Table 1 shows the preoperative characteristics of the patients. The preoperative characteristics were comparable except for statistically higher body mass index (BMI) in the cECLS group (BMI $\geq 30: 35 \%$ in cECLS $v$. $19 \%$ in pECLS, $\mathrm{P}=0.0079$ ), higher rate of previous cardiac surgery in the cECLS group ( $48 \%$ vs. $22 \%$ in pECLS, $\mathrm{P}=0.0001$ ), as well as higher $\mathrm{C}$-reactive protein (CRP) in the cECLS group $\{18$ [12-30] vs. 13 [6.7-24], $\mathrm{P}=0.013\}$. Further, norepinephrine was more in use in the cECLS group (58\% vs. $44 \%, \mathrm{P}=0.04)$ and the cardiopulmonary bypass time was significantly longer in the cECLS group $\{120 \min [78-180]$ vs. $99 \min [60-130], \mathrm{P}=0.0014\}$.

To adjust for differences between the groups, a 1:1 propensity score analysis was computed with univariate analysis assessing a predefined set of preoperative risk factors. The matching resulted in two matched groups (each 55 patients) based on the baseline characteristics of the patients (Figure 1). Table 2 shows the characteristics of the matched groups. The groups were well matched with no statistically significant differences between the matched groups. The cECLS group had median age of 54 years [48-60 years] vs. 54 years [43-60 years] in the pECLS group $(\mathrm{P}=0.67)$. The median duration of ECLS support was five days [two-seven days] in the cECLS group $v$ s. five days [three-ten days] in the pECLS group $(\mathrm{P}=0.39)$. The HeartWare HVAD pump was predominantly implanted in both groups ( $71 \%$ cECLS $v s .76 \%$ in pECLS, $\mathrm{P}=0.67$ ). Table 3 shows postoperative (after durable MCS implantation) outcome in the matched group of patients over the entire duration of follow up. Postoperative mechanical right ventricular support was necessary in 53\% of the cECLS patients $v s$. $44 \%$ of the pECLS patients $(\mathrm{P}=0.45)$. The amount of chest tube output in the first twenty-four hours and rate of re-exploration for bleeding 


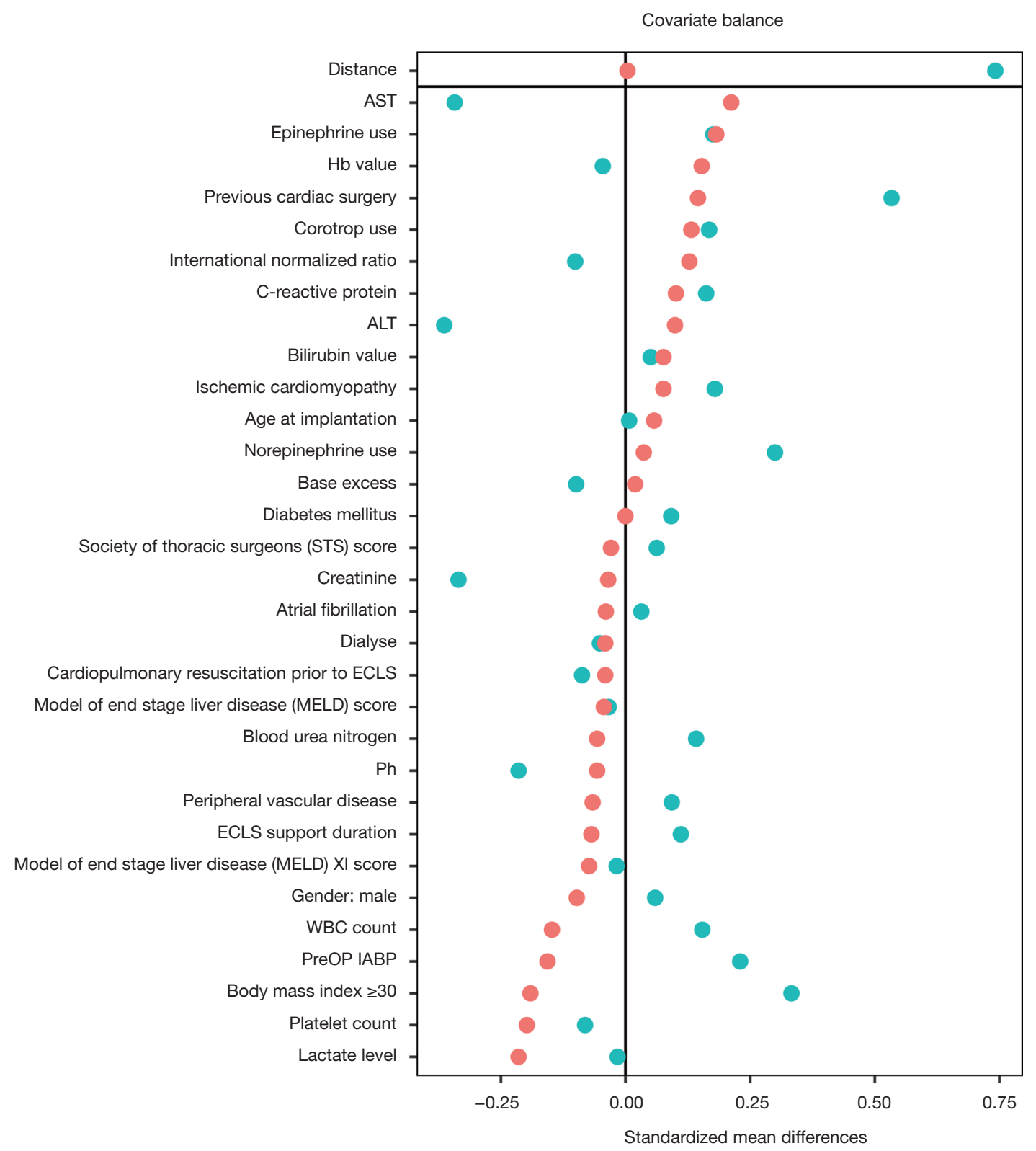

Figure 1 Bias reduction plotted as standardized mean differences of baseline variables between the central ECLS and peripheral ECLS groups. Baseline differences before propensity score matching (blue dots) were effectively balanced after matching (red dots). ECLS, extracorporeal life support.

were comparable between the groups. All other post durable MCS related complications were comparable between the groups. There was a slightly higher rate of pump thrombosis after durable MCS implantation in the cECLS group ( 0.15 events per patient year $v s .0 .06$ events per patient year). However, looking at the pump thrombosis events in the first year of follow up, no statistically significant difference was observed (Log-rank test, $\mathrm{P}=0.82$ ). Meanwhile, the rate of postoperative stroke was comparable between the groups $(27 \%$ in cECLS vs. $25 \%$ in pECLS, $\mathrm{P}=1.00)$. The median duration of the durable MCS support was 320 days [26-970 days] in cECLS group $v s .240$ days 
Table 1 Pre- and intraoperative characteristics of the overall population

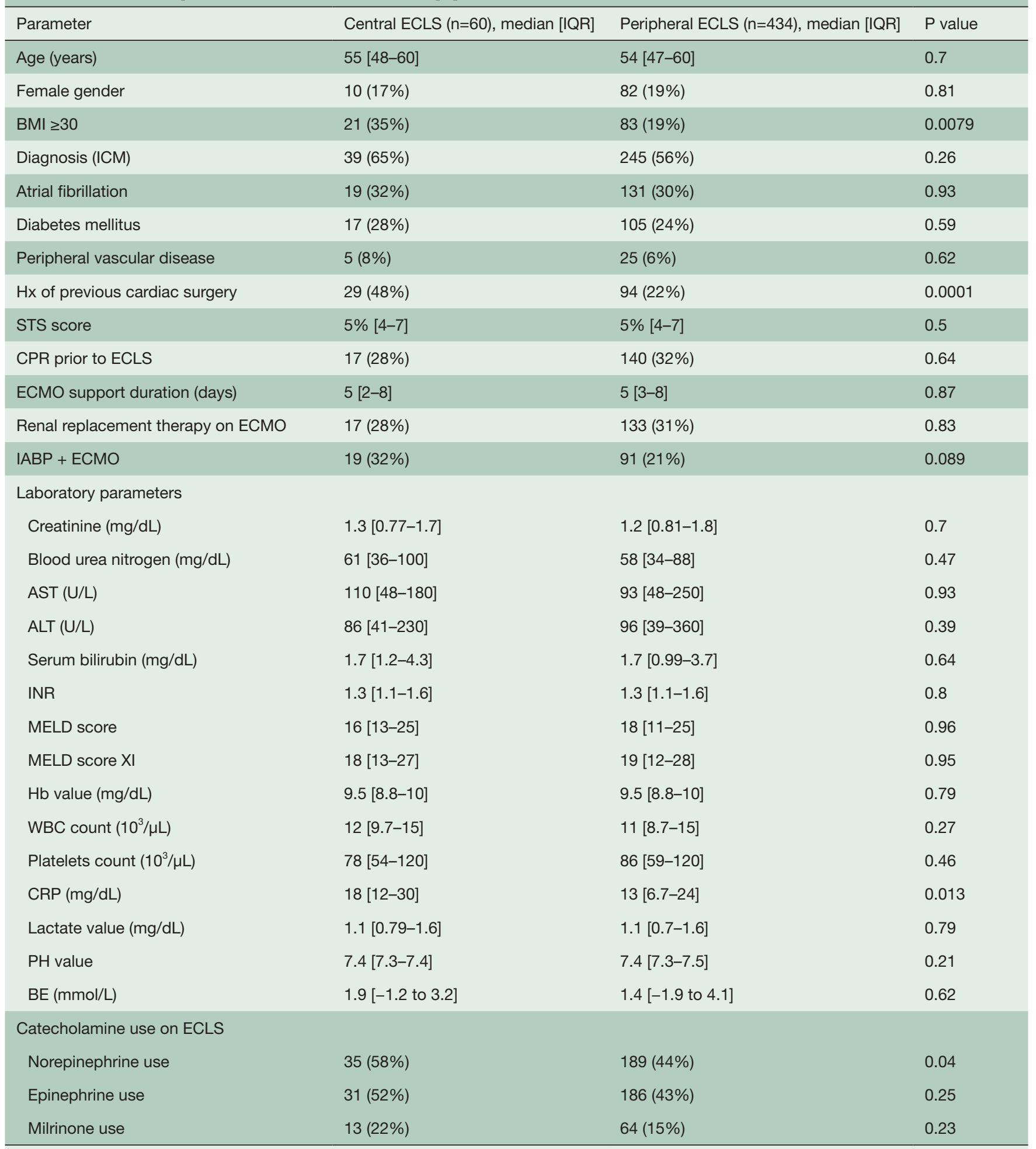

Table 1 (continued) 


\begin{tabular}{|c|c|c|c|}
\hline Parameter & Central ECLS $(n=60)$, median [IQR] & Peripheral ECLS $(n=434)$, median [IQR] & $P$ value \\
\hline \multicolumn{4}{|l|}{ Intraoperative parameters } \\
\hline CPB used for VAD implantation & $41(68 \%)$ & $247(57 \%)$ & 0.12 \\
\hline Less Invasive VAD implantation & $2(3 \%)$ & $40(9 \%)$ & 0.2 \\
\hline Total surgery time (min) & $250[190-340]$ & $240[190-320]$ & 0.7 \\
\hline CPB time (min) & $120[78-180]$ & $99[60-130]$ & 0.001 \\
\hline Concomitant procedures + VAD & $13(22 \%)$ & $81(19 \%)$ & 0.7 \\
\hline VAD type: HeartMate II & $10(17 \%)$ & $69(16 \%)$ & 1.00 \\
\hline VAD type: HeartMate III & $6(10 \%)$ & $39(9 \%)$ & 0.94 \\
\hline VAD type: HeartWare HVAD & $44(73 \%)$ & $326(75 \%)$ & 0.92 \\
\hline
\end{tabular}

[24-840 days] in the pECLS group $(\mathrm{P}=0.83)$. During the follow up, heart transplantation was performed in $20 \%$ of the cECLS group $v s .24 \%$ in the pECLS group $(\mathrm{P}=0.82)$. Further, up to $60 \%$ of the cECLS patients expired while on durable MCS vs. $55 \%$ of the pECLS patients $(\mathrm{P}=0.7)$. Figure 2 shows the Kaplan-Meier survival curve of the matched groups. There were no statistically significant differences in the short- and long-term outcomes between the groups ( $\log$ rank test, $\mathrm{P}=0.73$ ). The thirty-day and one-year mortality of the matched groups were $29 \%$ in cECLS group vs. $27 \%$ in pECLS group $(\mathrm{P}=1.00)$ and $49 \%$ in cECLS group vs. $49 \%$ in pECLS group ( $\mathrm{P}=1.00)$ respectively. No major differences in the outcome between the three types of pumps in the matched groups were observed. The thirty-day and one-year survival rates in the 39 matched patients in cECLS group supported with HeartWare HVAD were $72 \%$ and $49 \%$ respectively. This was comparable with the thirty-day and one-year survival rates of $70 \%$ and $60 \%$ in the 10 matched cECLS group supported with HeartMate II as well as the thirty-day and one-year survival rates of $67 \%$ and $67 \%$ in the 6 matched cECLS patients supported with HeartMate III pump.

\section{Discussion}

The recent years have witnessed a widespread use of ECLS systems for patients in cardiogenic shock $(7,8)$. In this multicentre study, we performed a sub analysis of the
Durable MCS after ECLS registry data to specifically investigate whether the preoperative ECLS implantation strategy has any impact on the outcome following durable MCS implantation. The findings of this study show that the outcome is comparable after durable MCS implantation in matched groups of patients regardless of the preoperative ECLS cannulation strategy. The postoperative MCS related morbidities of the matched groups were also comparable.

Durable MCS implantation and/or heart transplantation remains the only therapy option in otherwise viable patients on ECLS who fail to show adequate recovery of the ventricular function in absence of neurological deficits. Even though the new US allocation system now prioritizes patients waiting on ECLS, outcome after heart transplantation on ECLS remains poor $(9,10)$. Furthermore, considering issues of organ shortage and resulting extended waiting times in Europe for heart transplantation, timely transplantation may not be a realistic therapy option for patients on ECLS. Therefore, at least in Europe, durable MCS therapy remains the main treatment option for this patient population. In an effort to determine "the point of no return" in this patient population on ECLS, our group established the Durable MCS after ECLS registry, which includes data from 531 patients who underwent durable MCS implantation after ECLS support (4). In the first analysis of the registry data, we were able to show that the overall survival is very limited in this patient population and not comparable to the outcome in "traditional" MCS 
Table 2 Pre- and intraoperative characteristics of the patients after 1:1 propensity score matching

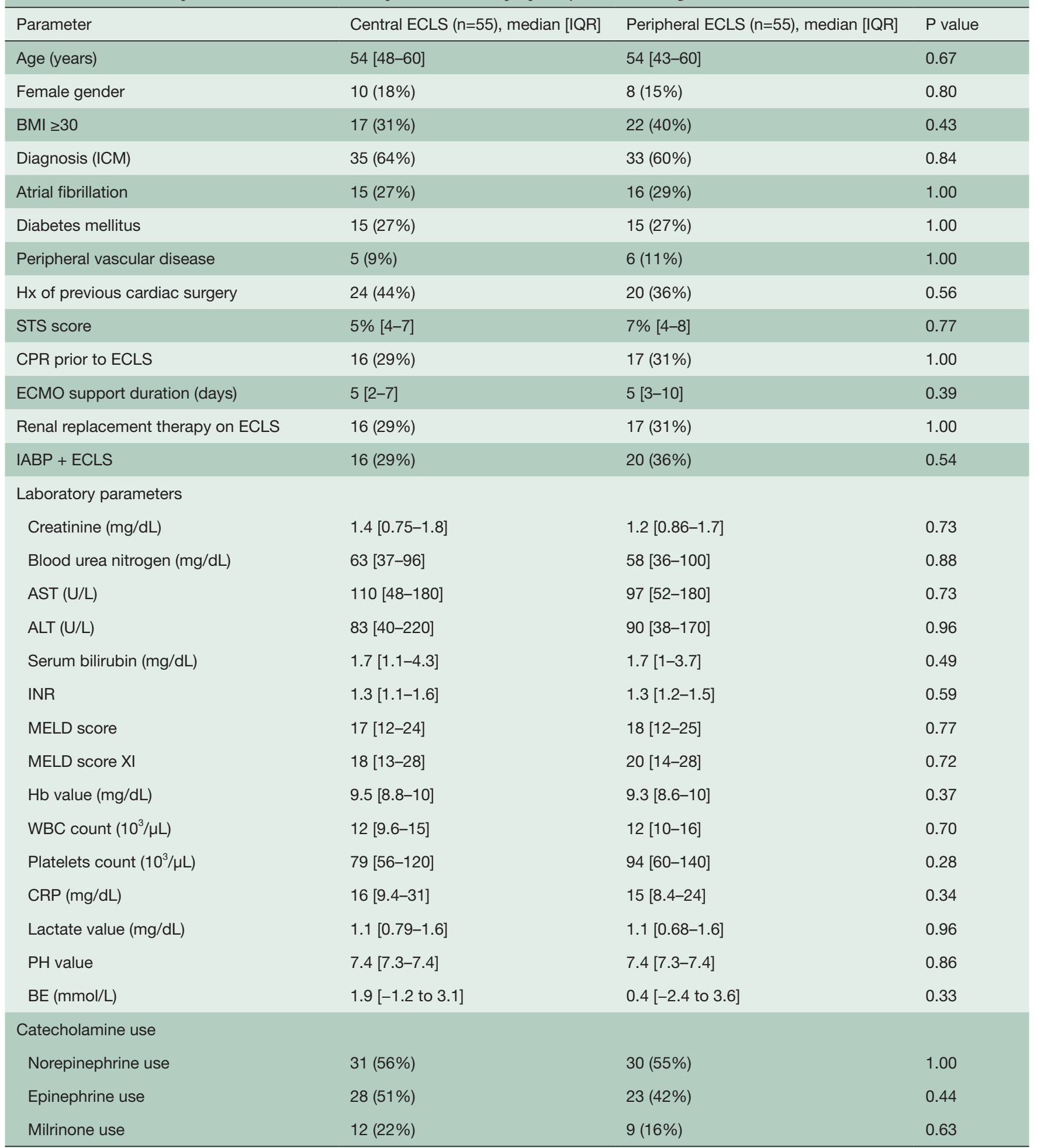

Table 2 (continued) 
Table 2 (continued)

\begin{tabular}{|c|c|c|c|}
\hline Parameter & Central ECLS $(n=55)$, median [IQR] & Peripheral ECLS ( $n=55)$, median [IQR] & $P$ value \\
\hline CPB used for VAD implantation & $38(69 \%)$ & $39(71 \%)$ & 1.00 \\
\hline Less invasive VAD implantation & $2(4 \%)$ & $3(5 \%)$ & 1.00 \\
\hline Total surgery time (min) & $250[190-340]$ & $250[210-330]$ & 0.67 \\
\hline Concomitant procedures + VAD & $13(24 \%)$ & $18(33 \%)$ & 0.40 \\
\hline VAD type: HeartMate II & $10(18 \%)$ & $10(18 \%)$ & 1.00 \\
\hline VAD type: HeartMate III & $6(11 \%)$ & $3(5 \%)$ & 0.49 \\
\hline VAD type: HeartWare HVAD & $39(71 \%)$ & $42(76 \%)$ & 0.67 \\
\hline
\end{tabular}

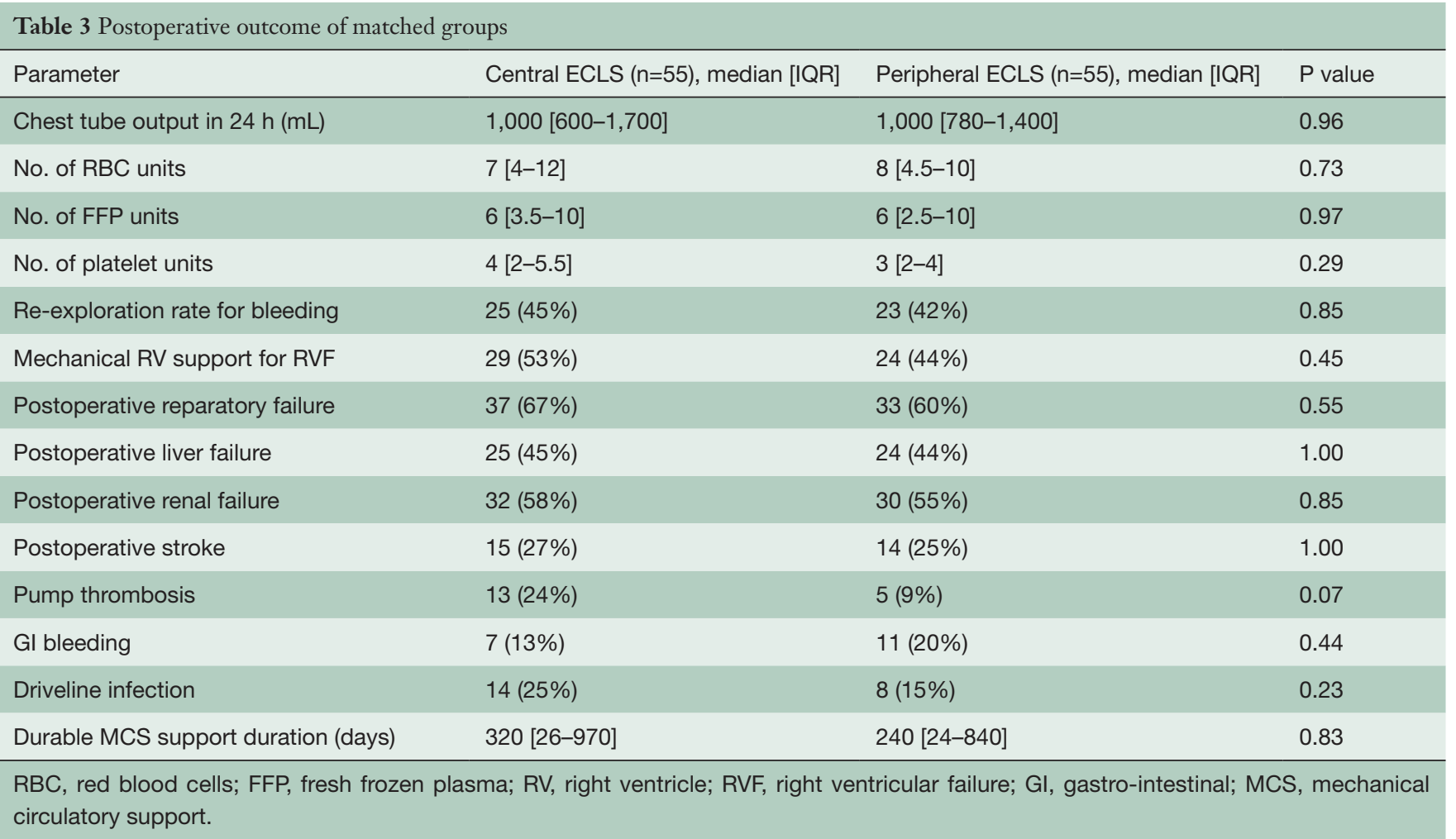

candidates. The postoperative morbidities, manifested as increased right ventricular failure and higher rate of postoperative complications, were also higher than the morbidities following durable MCS implantation in patients without ECLS support. Furthermore, we were able to find several predictors of one-year mortality in this patient 


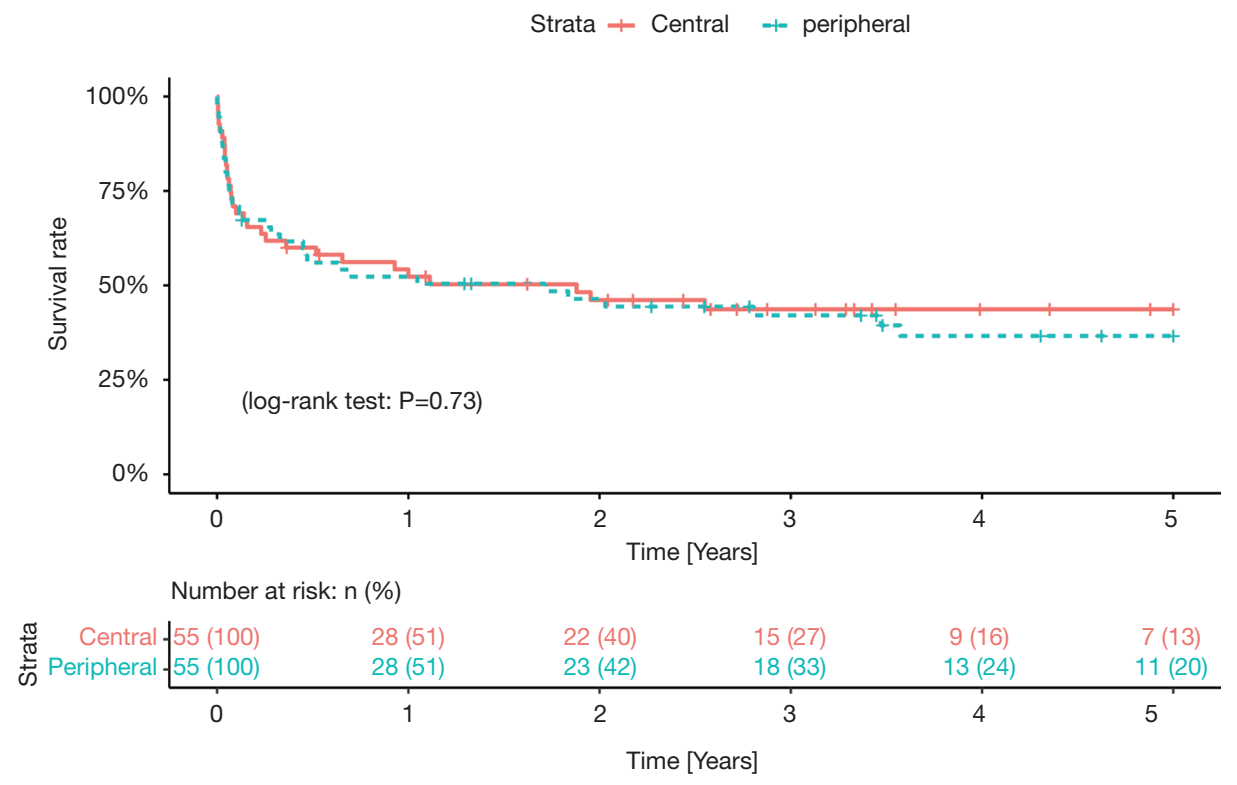

Figure 2 The Kaplan-Meier survival curves after durable MCS implantation in patients on ECLS. The thirty-day and one-year mortality of the matched groups were $29 \%$ in central ECLS group vs. $27 \%$ in peripheral ECLS group, and $49 \%$ in central ECLS group vs. $49 \%$ in peripheral ECLS group respectively $(\mathrm{P}=1.00$ each). The short and long-term survival rates were comparable between the matched groups (Log rank test, $\mathrm{P}=0.73$ ). ECLS, extra-corporeal life support; MCS, mechanical circulatory support.

population including; age, female gender, lactate value, high MELD XI score, history of atrial fibrillation, history of previous cardiac surgery and $\mathrm{BMI} \geq 30$. On the basis of this data, a formula to estimate one-year survival after durable MCS implantation and an app (durable MCS after ECLS calculator) were created to facilitate future candidate selection (4). The app may be downloaded for free from the Apple Store or the Google Play Store.

Patients requiring ECLS support may be cannulated using a femoro-femoral approach; the so called peripheral ECLS technique, or using aorta /subclavian artery for returning the oxygenated blood from ECLS circuit; the so called central ECLS approach. In patients with cECLS and aortic cannulation, direct cannulation of the aorta may be performed. Alternatively, a graft is anastomosed to the aorta and tunnelled allowing sternum closure (6). Each of these two cannulation strategies has its own advantages and disadvantages. Some centers prefer the central cannulation approach with the advantage of having direct flow stream from the outflow cannula into the aorta and the arch vessels, coronaries and the rest of the body. Other centers prefer the peripheral cannulation technique as it is faster, can be performed at the bed side, and is less invasive compared to the central cannulation technique. However, higher peripheral vascular complications have been reported in pECLS patients (11). There are many studies investigating the differences in outcome and post implantation complications in patients undergoing central or peripheral ECLS implantation. In a different study, the outcome of these two cannulation techniques was investigated in 37 patients (12). In that study, no particular oxygenation/ ventilation, hemodynamic, or end organ function advantage was observed with either cannulation technique. However, more bleeding and resternotomy complications were observed in cECLS patients. In another multicentre study of postcardiotomy patients supported with ECLS, Mariscalco et al. analysed the outcome of 781 patients receiving ECLS for postcardiotomy cardiogenic shock (5). The findings of that study showed higher in-hospital mortality in cECLS patients as well as higher reoperation rate for bleeding/ tamponade and blood transfusion requirements. All of the published studies so far questioned the impact of ECLS cannulation strategy on the outcome $(5,11,12)$. However, none of the published studies specifically investigate the 
impact of the preoperative ECLS cannulation strategy on the outcome following durable MCS implantation in patients on ECLS. The findings of this study show that the preoperative ECLS cannulation strategy has no impact on the postoperative outcome after durable MCS implantation. In contrary to our hypothesis, the more physiological form (cECLS strategy) showed no advantage with regard to postoperative morbidity and mortality.

The main limitation of this study is its retrospective nature. However, data in many centers were prospectively collected and entered in a corresponding data bank. Additionally, this was not a randomized study and despite matching our ECLS groups using several covariates, there may still be confounding by indication. Nevertheless, as shown in Figure 1, the groups were well matched and we therefore believe that the study outcome is representative. However, as the peripheral cannulation strategy dominated the study population, it may potentially introduce a hidden exclusion bias.

In conclusion, this study showed that the outcome after durable MCS implantation in matched groups of patients bridged with ECLS is similar regardless of the preoperative ECLS cannulation strategy. The implications of this study may discourage some clinicians from changing a well-functioning peripheral or central ECLS cannulation approach based on the incorrect notion that one approach may be superior to the other. Future research may need to focus on determining whether left ventricular pressures and risk of lung oedema in ECLS vary based on cannulation strategy.

\section{Acknowledgments}

Funding: None.

\section{Footnote}

Conflicts of Interest: The authors have no conflicts of interest to declare.

Open Access Statement: This is an Open Access article distributed in accordance with the Creative Commons Attribution-NonCommercial-NoDerivs 4.0 International License (CC BY-NC-ND 4.0), which permits the noncommercial replication and distribution of the article with the strict proviso that no changes or edits are made and the original work is properly cited (including links to both the formal publication through the relevant DOI and the license). See: https://creativecommons.org/licenses/by-nc-nd/4.0/.

\section{References}

1. Marasco SF, Lukas G, McDonald M, et al. Review of ECMO (extra corporeal membrane oxygenation) support in critically ill adult patients. Heart Lung Circ 2008;17 Suppl 4:S41-7.

2. Durinka JB, Bogar LJ, Hirose H, et al. End-organ recovery is key to success for extracorporeal membrane oxygenation as a bridge to implantable left ventricular assist device. ASAIO J 2014;60:189-92.

3. Maxhera B, Albert A, Ansari E, et al. Survival predictors in ventricular assist device patients with prior extracorporeal life support: selecting appropriate candidates. Artif Organs 2014;38:727-32.

4. Saeed D, Potapov E, Loforte A, et al. Transition From Temporary to Durable Circulatory Support Systems. J Am Coll Cardiol 2020;76:2956-64.

5. Mariscalco G, Salsano A, Fiore A, et al. Peripheral versus central extracorporeal membrane oxygenation for postcardiotomy shock: Multicenter registry, systematic review, and meta-analysis. J Thorac Cardiovasc Surg 2020;160:1207-1216.e44.

6. Saeed D, Maxhera B, Westenfeld R, et al. An Alternative Approach for Perioperative Extracorporeal Life Support Implantation. Artif Organs 2015;39:719-23.

7. Aubin H, Petrov G, Dalyanoglu H, et al. Four-year experience of providing mobile extracorporeal life support to out-of-center patients within a suprainstitutional network-Outcome of 160 consecutively treated patients. Resuscitation 2017;121:151-7.

8. Aubin H, Petrov G, Dalyanoglu H, et al. A Suprainstitutional Network for Remote Extracorporeal Life Support: A Retrospective Cohort Study. JACC Heart Fail 2016;4:698-708.

9. Barge-Caballero E, Almenar-Bonet L, GonzalezVilchez F, et al. Clinical outcomes of temporary mechanical circulatory support as a direct bridge to heart transplantation: a nationwide Spanish registry. Eur J Heart Fail 2018;20:178-86.

10. Fukuhara S, Takeda K, Kurlansky PA, et al. Extracorporeal membrane oxygenation as a direct bridge to heart transplantation in adults. J Thorac Cardiovasc Surg 
2018;155:1607-1618.e6.

11. Ranney DN, Benrashid E, Meza JM, et al. Central Cannulation as a Viable Alternative to Peripheral Cannulation in Extracorporeal Membrane Oxygenation. Semin Thorac Cardiovasc Surg 2017;29:188-95.

Cite this article as: Saeed D, Potapov E, Loforte A, Morshuis M, Schibilsky D, Zimpfer D, Riebandt J, Pappalardo F, Attisani M, Rinaldi M, Pacini D, Haneya A, Ramjankhan F, Donker DW, Jorde UP, Otto W, Stein J, Tsyganenko D, Al-Naamani A, Wieloch R, Ayala R, Cremer J, Borger M, Lichtenberg A, Gummert J. Impact of extra-corporeal life support (ECLS) cannulation strategy on outcome after durable mechanical circulation support system implantation on behalf of durable MCS after ECLS Study Group. Ann Cardiothorac Surg 2021;10(3):353-363. doi: 10.21037/acs-2020-cfmcs-251
12. Saeed D, Stosik H, Islamovic M, et al. Femoro-femoral versus atrio-aortic extracorporeal membrane oxygenation: selecting the ideal cannulation technique. Artif Organs 2014;38:549-55. 